\title{
ANALOGOUS OF CONVERSE PALEY-WIENER THEOREM IN THE CONTEXT OF POSITIVE DEFINITE KERNEL
}

\author{
Osmin Ferrer $^{1} \S$, Arnaldo de la Barrera ${ }^{2}$, \\ Jorge Rodriguez ${ }^{3}$ \\ ${ }^{1}$ Department of Mathematics, University of Sucre \\ Sincelejo - 700001, COLOMBIA \\ 2 Department of Mathematics, University of Pamplona \\ Pamplona - 543050, COLOMBIA \\ 3 Department of Mathematics \\ University of Atlantico - University of Norte \\ Barranquilla - 08002, COLOMBIA
}

\begin{abstract}
We formulate and prove an analogous of converse Paley-Wiener theorem in the context of positive definite kernel. The main ingredient in the proof is Kolmogorov decomposition. Finally, some applications to stochastic processes are given.
\end{abstract}

AMS Subject Classification: 53C2, 53C42, 42A82, 60G10

Key Words: positive definite kernels; Paley-Wiener theorem; Kolgomorov decomposition

\section{Paley-Wiener Theorem}

The fundamental criterium of stability, and historically the first one, is due to Paley and Wiener [4]. It is based on the known fact that a linear bounded operator $T$ on a Banach space is invertible if

$$
\|I-T\|<1 .
$$

Received: September 23, 2020

(C) 2021 Academic Publications

${ }^{\S}$ Correspondence author 
Theorem 1. (Paley-Wiener) Let $\left\{x_{n}\right\}_{n \in \mathbb{N}}$ be a basis in the Banach space $X$, and suppose that $\left\{y_{n}\right\}_{n \in \mathbb{N}}$ is a sequence of elements of $X$ such that

$$
\left\|\sum_{n=1}^{N} c_{n}\left(x_{n}-y_{n}\right)\right\| \leq \lambda\left\|\sum_{n=1}^{N} c_{n} x_{n}\right\|
$$

for all $N \in \mathbb{N}$, some constant $\lambda$, with $0 \leq \lambda<1$ and for any sequence of scalars $\left\{c_{n}\right\}_{n \in \mathbb{N}}$. Then $\left\{y_{n}\right\}_{n \in \mathbb{N}}$ is a basis for $X$ equivalent to $\left\{x_{n}\right\}_{n \in \mathbb{N}}$.

See [5, Theorem 10] for a proof.

The following lemmas are well known, see [5] and [3].

Lemma 2. (Polar Decomposition) Every bounded invertible operator $T$ on Hilbert space can be factored in the form $T=U P$, where $U$ is a unitary operator and $P$ is a positive operator.

Lemma 3. If $T$ is a bounded self-adjoint operator on Hilbert space, then

$$
\|T\|=\sup \left\{\frac{\langle T f, f\rangle}{\|f\|^{2}}: f \neq 0\right\} .
$$

In what follows we present the reciprocal of Paley-Wiener theorem, see [5] and $[3]$.

If a set $\left\{x_{n}\right\}$ approximately satisfies Parseval's formula then there exists at least one orthonormal set which it is near.

Theorem 4. (Duffin and Eachus) Let $\left\{y_{n}\right\}$ be a sequence of elements in Hilbert space and let $\theta$ be a number such that $0 \leq \theta<1$. Suppose that

$$
(1-\theta)^{2} \sum_{i=1}^{n}\left|a_{i}\right|^{2} \leq\left\|\sum_{i=1}^{n} a_{i} y_{i}\right\|^{2} \leq(1+\theta)^{2} \sum_{i=1}^{n}\left|a_{i}\right|^{2}
$$

for each finite scalars $a_{1}, a_{2}, \ldots, a_{n}$. Then there exists an orthonormal set of vectors $\left\{x_{n}\right\}$ such that

$$
\left\|\sum_{i=1}^{n} a_{i}\left(x_{i}-y_{i}\right)\right\| \leq \theta\left(\sum_{i=1}^{n}\left|a_{i}\right|^{2}\right)^{1 / 2}
$$

for each finite scalars $a_{1}, a_{2}, \ldots, a_{n}$.

See [5, Theorem 10] and [3] for a proof. 


\section{Kolmogorov decomposition theorem}

\subsection{The Hilbert space associated to a positive definite operator valued kernel}

Let $\left\{\mathcal{H}_{n}\right\}_{n \in \mathbb{Z}}$ be a family of Hilbert spaces. An operator valued kernel on $\mathbb{Z}$ to $\left\{\mathcal{H}_{n}\right\}_{n \in \mathbb{Z}}$ is an application $K: \mathbb{Z} \times \mathbb{Z} \rightarrow \bigcup_{m, n \in \mathbb{Z}} \mathcal{L}\left(\mathcal{H}_{m}, \mathcal{H}_{n}\right)$ such that

$$
K(n, m) \in \mathcal{L}\left(\mathcal{H}_{m}, \mathcal{H}_{n}\right) \quad \text { for } n, m \in \mathbb{Z}
$$

In this section and the following one, unless it is otherwise stated, all the kernels will be operator valued ones.

A sequence $\left\{h_{n}\right\}$ in $\oplus_{n \in \mathbb{Z}} \mathcal{H}_{n}$ is said to have finite support if $h_{n}=0$ except for a finite number of integers $n$.

A kernel $K$ on $\mathbb{Z}$ to $\left\{\mathcal{H}_{n}\right\}_{n \in \mathbb{Z}}$ is a positive definite kernel if

$$
\sum_{n, m \in \mathbb{Z}}\left\langle K(n, m) h_{m}, h_{n}\right\rangle_{\mathcal{H}_{n}} \geq 0
$$

for every sequence $\left\{h_{n}\right\}$ in $\oplus_{n \in \mathbb{Z}} \mathcal{H}_{n}$ with finite support.

Let $K$ be a positive definite kernel. Let $\mathcal{F}$ be the linear space of elements $\bigoplus_{n \in \mathbb{Z}} \mathcal{H}_{n}$ and $\mathcal{F}_{o}$ be the space of elements in $\mathcal{F}$ with finite support.

Define $B_{K}: \mathcal{F}_{O} \times \mathcal{F}_{o} \rightarrow \mathbb{C}$ with

$$
B_{K}(f, g)=\sum_{m, n \in \mathbb{Z}}\left\langle K(n, m) f_{m}, g_{n}\right\rangle_{\mathcal{H}_{n}},
$$

for $f, g \in \mathcal{F}_{o}, f=\left\{f_{n}\right\}, g=\left\{g_{n}\right\}, f_{n}, g_{n} \in \mathcal{H}_{n}$.

Note that $B_{K}$ satisfies all the properties of an inner product, except for the fact that the set

$$
\mathcal{N}_{K}=\left\{h \in \mathcal{F}_{o}: B_{K}(h, h)=0\right\},
$$

could be non-trivial.

According to the Cauchy-Schwarz inequality

$$
\mathcal{N}_{K}=\left\{h \in \mathcal{F}_{o}: B_{K}(h, g)=0, \text { for all } g \in \mathcal{F}_{o}\right\}
$$

hence $\mathcal{N}_{K}$ is a linear subspace of $\mathcal{F}_{o}$.

The quotient space $\mathcal{F}_{o} / \mathcal{N}_{K}$ is also a linear subspace. If $[h]$ stands for the class of the element $h$ in $\mathcal{F}_{o} / \mathcal{N}_{K}$, then the application

$$
\langle[h],[g]\rangle=B_{K}(h, g), \quad h, g \in \mathcal{F}_{o},
$$


is well defined. To prove that $\langle\cdot, \cdot\rangle$ is an inner product on $\mathcal{F}_{o} / \mathcal{N}_{K}$ is straightforward.

The completion of $\mathcal{F}_{o} / \mathcal{N}_{K}$ with respect to the norm induced by this inner product is a Hilbert space. It is known as the Hilbert space associated to the positive definite kernel $K$ and it is denoted by $\mathcal{H}_{K}$. The inner product and the norm of $\mathcal{H}_{K}$ will be represented as $\langle\cdot, \cdot\rangle_{\mathcal{H}_{K}}$ and $\|\cdot\|_{\mathcal{H}_{K}}$ respectively. This norm will be named as the norm induced by $K$.

\subsection{Kolmogorov Decomposition Theorem}

The following theorem is a version of the classic result of Kolmogorov (see [1] for a historical review).

Theorem 5. (Kolmogorov) Let $K$ be a positive definite kernel. Then there exists a Hilbert space $\mathcal{H}_{K}$ and a map $V$ defined on $\mathbb{Z}$ such that $V(n)$ belongs to $\mathcal{L}\left(\mathcal{H}_{n}, \mathcal{H}_{K}\right)$ for each $n \in \mathbb{Z}$ and

(a) $K(n, m)=V^{*}(n) V(m)$ if $n, m \in \mathbb{Z}$.

(b) $\mathcal{H}_{K}=\bigvee_{n \in \mathbb{Z}} V(n) \mathcal{H}_{n}$.

(c) The decomposition is unique in the following sense: if $\mathcal{H}^{\prime}$ is another Hilbert space and $V^{\prime}$ defined on $\mathbb{Z}$ is an application such that $V^{\prime}(n) \in$ $\mathcal{L}\left(\mathcal{H}_{n}, \mathcal{H}_{K}\right)$ for each $n \in \mathbb{Z}$ that satisfies (a) and (b), then there exists a unitary operator $\Phi: \mathcal{H}_{K} \rightarrow \mathcal{H}^{\prime}$ such that $\Phi V(n)=V^{\prime}(n)$ for all $n \in \mathbb{Z}$.

A proof of this theorem can be found in [6, Theorem 3.1].

An application $V$ that satisfies the property (a) in Theorem 5 will be called the Kolmogorov Decomposition of the Kernel $K$ or simply, a Decomposition of the kernel $K$ (see [6]). The property (b) is referred to as the minimality property of Kolmogorov Decomposition. The meaning of property (c) is that, under the minimality condition (b), the Kolmogorov decomposition is essentially unique.

The coming result is our version of Theorem 4. This result is related with Lemmas 2, 3 and the Kolmogorov decomposition.

Theorem 6. Let $K: \mathbb{Z} \times \mathbb{Z} \rightarrow \mathcal{L}(\mathcal{H})$ be a positive definite kernel. Let $K_{1}: \mathbb{Z} \times \mathbb{Z} \rightarrow \mathcal{L}(\mathcal{H})$ be a positive definite kernel and $\theta$ be a number such that 
$0 \leq \theta<1$. Suppose that

$$
\begin{aligned}
(1-\theta)^{2} \sum_{n, m \in \mathbb{Z}}\left\langle K_{1}(n, m) h_{m}, h_{n}\right\rangle_{\mathcal{H}} & \leq \sum_{n, m \in \mathbb{Z}}\left\langle K_{2}(n, m) h_{m}, h_{n}\right\rangle_{\mathcal{H}} \\
& \leq(1+\theta)^{2} \sum_{n, m \in \mathbb{Z}}\left\langle K_{1}(n, m) h_{m}, h_{n}\right\rangle_{\mathcal{H}}
\end{aligned}
$$

for all sequences $\left\{h_{n}\right\}_{n \in \mathbb{Z}} \subset \mathcal{H}$ with finite support. Then there exists a sequence $\left\{g_{n}\right\}_{n \in \mathbb{Z}} \subset \mathcal{H}_{K}$ for all $n \in \mathbb{Z}$ such that

$$
\left\|\sum_{n \in \mathbb{Z}}\left(g_{n}-V_{K}(n)\right) h_{n}\right\|_{\mathcal{H}_{K}} \leq \theta\left\|\sum_{n \in \mathbb{Z}} V_{K_{1}}(n) h_{n}\right\|_{\mathcal{H}_{K_{1}}},
$$

for any sequence $\left\{h_{n}\right\}_{n \in \mathbb{Z}} \subset \mathcal{H}$ with finite support.

Proof. On the one hand let us define a mapping $G: \mathcal{H}_{K_{1}} \rightarrow \mathcal{H}_{K}$ by

$$
G\left(\sum_{n \in \mathbb{Z}} V_{K_{1}}(n) h_{n}\right)=\sum_{n \in \mathbb{Z}} V_{K}(n) h_{n}
$$

where $\left\{h_{n}\right\}_{n \in \mathbb{Z}}$ is a sequence with finite support in $\mathcal{H}$.

On the other hand, since $K$ and $K_{1}$ are positive definite kernels by the Kolmogorov decomposition theorem we have

$$
K(n, m)=V_{K}^{*}(n) V_{K}(m), \quad m, n \in \mathbb{Z}
$$

and

$$
K_{1}(n, m)=V_{K_{1}}^{*}(n) V_{K_{1}}(m), \quad m, n \in \mathbb{Z} .
$$

Taking into account the above expressions

$$
\begin{aligned}
& \sum_{m, n \in \mathbb{Z}}\left\langle K(n, m) h_{m}, h_{n}\right\rangle_{\mathcal{H}}=\sum_{m, n \in \mathbb{Z}}\left\langle V_{K}(n)^{*} V_{K}(m) h_{m}, h_{n}\right\rangle_{\mathcal{H}} \\
& =\left\langle\sum_{m \in \mathbb{Z}} V_{K}(m) h_{m}, \sum_{n \in \mathbb{Z}} V_{K}(n) h_{n}\right\rangle_{\mathcal{H}_{K}}=\left\|\sum_{n \in \mathbb{Z}} V_{K}(n) h_{n}\right\|_{\mathcal{H}_{K}}^{2} .
\end{aligned}
$$

Analogously,

$$
\sum_{m, n \in \mathbb{Z}}\left\langle K_{1}(n, m) h_{m}, h_{n}\right\rangle_{\mathcal{H}}=\left\|\sum_{n \in \mathbb{Z}} V_{K_{1}}(n) h_{n}\right\|_{\mathcal{H}_{K_{1}}}^{2} .
$$


Thus, if $f=\sum_{n \in \mathbb{Z}} V_{K_{1}}(n) h_{n}$ we may express relation (2) in the form

$$
(1-\theta)\|f\|_{\mathcal{H}_{K_{1}}} \leq\|G f\|_{\mathcal{H}_{K}} \leq(1+\theta)\|f\|_{\mathcal{H}_{K_{1}}}
$$

This shows, in particular, that $G$ is bounded from below. Since $\mathcal{H}_{K}=\bigvee_{n \in \mathbb{Z}} V_{K}(n) \mathcal{H}$, we have $\left\{V_{K}(n) \mathcal{H}\right\}_{n \in \mathbb{Z}}$ is complete. Then $G$ has a dense range and so must be invertible.

From the above results it is obtained that the mapping $G$ satisfies the conditions of Lemma 2, so $G=U P$. From (4) it follows that

$$
(1-\theta)\|f\|_{\mathcal{H}_{K_{1}}} \leq\|P f\|_{\mathcal{H}_{K}} \leq(1+\theta)\|f\|_{\mathcal{H}_{K_{1}}} \quad \text { whenever } \quad f \in \mathcal{H}_{K_{1}} \text {. }
$$

Since $P$ is a bounded self-adjoint operator on Hilbert space $\mathcal{H}_{K_{1}}$ by Lemma 3 ,

$$
(1-\theta)\langle f, f\rangle_{\mathcal{H}_{K_{1}}} \leq\langle P f, f\rangle_{\mathcal{H}_{K_{1}}} \leq(1+\theta)\langle f, f\rangle_{\mathcal{H}_{K_{1}}} .
$$

This may be written as

$$
-\theta\langle f, f\rangle_{\mathcal{H}_{K_{1}}} \leq\langle P f-f, f\rangle_{\mathcal{H}_{K_{1}}} \leq \theta\langle f, f\rangle_{\mathcal{H}_{K_{1}}}
$$

Since

$$
\langle P f-f, f\rangle_{\mathcal{H}_{K_{1}}}=\langle f, P f-f\rangle_{\mathcal{H}_{K_{1}}},
$$

it follows that $P-I$ is a self-adjoint bounded operator on Hilbert space $\mathcal{H}_{K_{1}}$, by Lemma 3, we have

$$
\|f-P f\|_{\mathcal{H}_{K_{1}}} \leq \theta\|f\|_{\mathcal{H}_{K_{1}}} \quad \text { for all } \quad f .
$$

Define $g_{n}=U V_{K_{1}}(n)$ for $n \in \mathbb{Z}$. Moreover,

$$
\begin{aligned}
\left\|\sum_{n \in \mathbb{Z}}\left(g_{n}-V_{K}(n)\right) h_{n}\right\|_{\mathcal{H}_{K}} & =\|U(f-P f)\|_{\mathcal{H}_{K}}=\|f-P f\|_{\mathcal{H}_{K}} \\
& \leq \theta\|f\|_{\mathcal{H}_{K_{1}}}=\theta\left\|\sum_{n \in \mathbb{Z}} V_{K_{1}}(n) h_{n}\right\|_{\mathcal{H}_{K_{1}}} .
\end{aligned}
$$




\section{Applications to Stochastic Processes}

\subsection{Multivariate Stochastic Processes}

In this section it will be used the decomposition of the covariance Kernels of the stochastic processes (see [6], Section 1, Chapter 6).

Definition 7. A pair $[\mathcal{K}, X]$, where $\mathcal{K}$ is a Hilbert space and $X=\left\{X_{n}\right\}_{n \in \mathbb{Z}}$ is a family of operators $X_{n}$ in $\mathcal{L}\left(\mathcal{H}_{n}, \mathcal{K}\right)$, is called a geometric model of the multivariate process with correlation kernel $K$, if

$$
K(m, n)=X_{m}^{*} X_{n}
$$

The Kolmogorov Decomposition Theorem shows that given a positive definite kernel $K$, there exists a geometric model of the multivariate process with correlation kernel $K$. If $[\mathcal{K}, X]$ is the geometric model of the multivariate process with covariance kernel $K$ then $\mathcal{H}_{X}$ will be the subspace of $\mathcal{K}$ generated for this model, that is,

$$
\mathcal{H}_{X}=\bigvee_{n \in \mathbb{Z}} X_{n} \mathcal{H}_{n}
$$

If $\left[\mathcal{K}^{\prime}, X^{\prime}\right]$ is another geometric model of the same process, then the Kolmogorov Decomposition Theorem guarantees the existence of an unitary operator $\Phi$ : $\mathcal{H}_{X} \rightarrow \mathcal{H}_{X^{\prime}}$ such that $\Phi X_{n}=X_{n}^{\prime}$ for all $n \in \mathbb{Z}$. This means that the geometry of the process is essentially determined by the choice of a geometric model such that

$$
\mathcal{K}=\bigvee_{n \in \mathbb{Z}} X_{n} \mathcal{H}_{n}
$$

Theorem 8. (Isomorphism) Let $[\mathcal{W}, X]$ be the geometric model of a multivariate process and let $K: \mathbb{Z} \times \mathbb{Z} \rightarrow \mathcal{L}(\mathcal{H})$ be the kernel of covariance associated with the process. Then there exists an unit operator $\Phi: \mathcal{H}_{K} \rightarrow \mathcal{H}_{X}$ such that

$$
\Phi V_{K}(n)=X_{n} \quad \text { for all } \quad n \in \mathbb{Z} \text {. }
$$

Theorem 9. Let $[\mathcal{W}, Y]$ be a geometrical model of a multivariate stochastic process. Let $[\mathcal{K}, X]$ be a geometrical model of a multivariate stochastic process and $\theta$ be a number such that $0 \leq \theta<1$. Suppose that

$$
(1-\theta)\left\|\sum_{n \in \mathbb{Z}} X_{n} h_{n}\right\|_{\mathcal{H}_{X}} \leq\left\|\sum_{n \in \mathbb{Z}} Y_{n} h_{n}\right\|_{\mathcal{H}_{Y}} \leq(1+\theta)\left\|\sum_{n \in \mathbb{Z}} X_{n} h_{n}\right\|_{\mathcal{H}_{X}},
$$


for all sequences $\left\{h_{n}\right\}_{n \in \mathbb{Z}} \subset \mathcal{H}$ with finite support. Then there exists a sequence $\left\{j_{n}\right\}_{n \in \mathbb{Z}} \subset \mathcal{H}_{Y}$ for all $n \in \mathbb{Z}$ such that

$$
\left\|\sum_{n \in \mathbb{Z}}\left(j_{n}-Y_{n}\right) h_{n}\right\|_{\mathcal{H}_{Y}} \leq \theta\left\|\sum_{n \in \mathbb{Z}} X_{n} h_{n}\right\|_{\mathcal{H}_{X}}
$$

for any sequence finite support $\left\{h_{n}\right\}_{n \in \mathbb{Z}} \subset \mathcal{H}$.

Proof. Let $K$ and $K_{1}$ be two kernels of covariance associated with the processes $Y=\left\{Y_{n}\right\}_{n \in \mathbb{Z}}$ and $X=\left\{X_{n}\right\}_{n \in \mathbb{Z}}$, respectively.

Let us consider the operators $\Phi_{1}: \mathcal{H}_{K} \rightarrow \mathcal{H}_{Y}$ such that

$$
\Phi_{1} V_{K}(n)=Y_{n} \quad \text { for all } \quad n \in \mathbb{Z}
$$

and $\Phi_{2}: \mathcal{H}_{K_{1}} \rightarrow \mathcal{H}_{X}$ such that

$$
\Phi_{2} V_{K_{1}}(n)=X_{n} \quad \text { for all } \quad n \in \mathbb{Z} .
$$

From above and the hypothesis it follows

$$
\begin{gathered}
(1-\theta)^{2}\left\|\sum_{n \in \mathbb{Z}} \Phi_{2} V_{K_{1}}(n) h_{n}\right\|_{\mathcal{H}_{X}}^{2} \leq\left\|\sum_{n \in \mathbb{Z}} \Phi_{1} V_{K}(n) h_{n}\right\|_{\mathcal{H}_{Y}}^{2} \\
\leq(1+\theta)^{2}\left\|\sum_{n \in \mathbb{Z}} \Phi_{2} V_{K_{1}}(n) h_{n}\right\|_{\mathcal{H}_{X}}^{2},
\end{gathered}
$$

for a number $\theta$ such that $0 \leq \theta<1$ and for all sequence finite support $\left\{h_{n}\right\}_{n \in \mathbb{Z}} \subset$ $\mathcal{H}$. Since

$$
\left\|\sum_{n \in \mathbb{Z}} \Phi_{2} V_{K_{1}}(n) h_{n}\right\|_{\mathcal{H}_{X}}^{2}=\left\|\sum_{n \in \mathbb{Z}} V_{K_{1}}(n) h_{n}\right\|_{\mathcal{H}_{K_{1}}}^{2}=\sum_{n, m \in \mathbb{Z}}\left\langle K_{1}(n, m) h_{m}, h_{n}\right\rangle_{\mathcal{H}},
$$

and

$$
\left\|\sum_{n \in \mathbb{Z}} \Phi_{1} V_{K}(n) h_{n}\right\|_{\mathcal{H}_{X}}^{2}=\sum_{n, m \in \mathbb{Z}}\left\langle K(n, m) h_{m}, h_{n}\right\rangle_{\mathcal{H}} .
$$

By Theorem 6, there exists a sequence $\left\{g_{n}\right\}_{n \in \mathbb{Z}} \subset \mathcal{H}_{K}$ for all $n \in \mathbb{Z}$ such that

$$
\left\|\sum_{n \in \mathbb{Z}}\left(g_{n}-V_{K}(n)\right) h_{n}\right\|_{\mathcal{H}_{K}} \leq \theta\left\|\sum_{n \in \mathbb{Z}} V_{K_{1}}(n) h_{n}\right\|_{\mathcal{H}_{K_{1}}},
$$


for any sequence $\left\{h_{n}\right\}_{n \in \mathbb{Z}} \subset \mathcal{H}$ with finite support.

Taking $j_{n}=\Phi_{1} g_{n}$ for all $n \in \mathbb{Z}$ we have

$$
\begin{aligned}
\left\|\sum_{n \in \mathbb{Z}}\left(j_{n}-Y_{n}\right) h_{n}\right\|_{\mathcal{H}_{Y}} & =\left\|\sum_{n \in \mathbb{Z}}\left(g_{n}-V_{K}(n)\right) h_{n}\right\|_{\mathcal{H}_{K}} \leq \theta\left\|\sum_{n \in \mathbb{Z}} V_{K_{1}}(n) h_{n}\right\|_{\mathcal{H}_{K_{1}}} \\
& =\theta\left\|\sum_{n \in \mathbb{Z}} \Phi_{2}^{-1} X_{n} h_{n}\right\|_{\mathcal{H}_{K_{1}}}=\theta\left\|\sum_{n \in \mathbb{Z}} X_{n} h_{n}\right\|_{\mathcal{H}_{X}},
\end{aligned}
$$

for any sequence $\left\{h_{n}\right\}_{n \in \mathbb{Z}} \subset \mathcal{H}$ with finite support.

\section{References}

[1] D.E. Evans and J.T. Lewis, Dilations of Irreversible Evolutions in Algebraic Quantum Theory, Communications of the Dublin Institute for Advanced Studies, Ser. A: Theoretical Physics, Vol. 24 (1977).

[2] R. Bruzual, A. De la Barrera and M. Domínguez, On positive definite kernels, related problems and applications, Extracta Math., 29, No 1-2 (2014), 97-115.

[3] R.J. Duffin and J.J. Eachus, Some notes on an expansion theorem of Paley and Wiener, Bull. Amer. Math. Soc., 48, No 12 (1942), 850-855.

[4] R. Paley and N. Wiener, Fourier Transforms in the Complex Domain, Reprint of the 1934 original, Amer. Math. Soc. Colloquium Publications, 19. Amer. Math. Soc., Providence, RI (1987).

[5] R.M. Young, An Introduction to Nonharmonic Fourier Series, Pure and Applied Mathematics, Vol. 93, Academic Press, Inc. [Harcourt Brace Jovanovich, Publ.], New York-London (1980).

[6] T. Constantinescu, Schur Parameters, Factorization and Dilation Problems, Operator Theory: Advances and Applications, 82, BirkhäuserVerlag, Basel (1996). 
